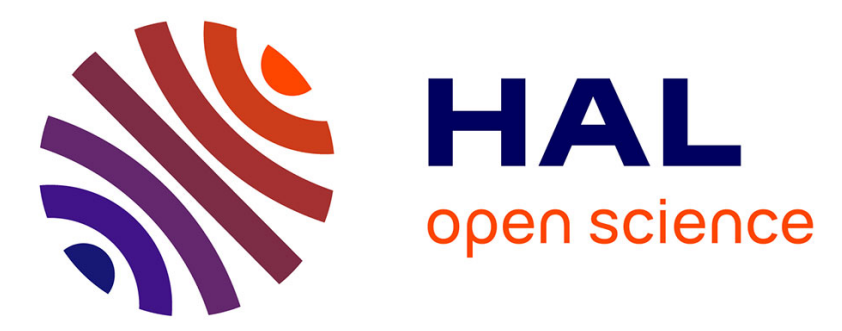

\title{
energy saving in existing buildings by an intelligent use of interoperable ICTs
}

\author{
Anna Osello, Andrea Acquaviva, Chiara Aghemo, Laura Blaso, Daniele \\ Dalmasso, David Erba, Giovanni Vincenzo Fracastoro, Damien Gondre, \\ Marco Jahn, Enrico Macii, et al.
}

\section{To cite this version:}

Anna Osello, Andrea Acquaviva, Chiara Aghemo, Laura Blaso, Daniele Dalmasso, et al.. energy saving in existing buildings by an intelligent use of interoperable ICTs. Energy efficiency, 2013, 6 (4), pp.707-723. hal-00985275

\section{HAL Id: hal-00985275 \\ https://hal.science/hal-00985275}

Submitted on 28 May 2014

HAL is a multi-disciplinary open access archive for the deposit and dissemination of scientific research documents, whether they are published or not. The documents may come from teaching and research institutions in France or abroad, or from public or private research centers.
L'archive ouverte pluridisciplinaire HAL, est destinée au dépôt et à la diffusion de documents scientifiques de niveau recherche, publiés ou non, émanant des établissements d'enseignement et de recherche français ou étrangers, des laboratoires publics ou privés. 
Energy saving in existing buildings by an intelligent use of interoperable ICTs

Anna Osello, Associate Professor, Politecnico di Torino, DISEG, Corso Duca degli Abruzzi 24, 10129 Torino (Italy) anna.osello@polito.it, +303351800784,+390110905335.

Andrea Acquaviva, Assistant Professor, Politecnico di Torino, DAUIN, Corso Duca degli Abruzzi 24, 10129 Torino (Italy). Chiara Aghemo, Full Professor, Politecnico di Torino, DENERG, Corso Duca degli Abruzzi 24, 10129 Torino (Italy).

Laura Blaso, PhD, Grant Researcher, Politecnico di Torino, DENER, Corso Duca degli Abruzzi 24, 10129 Torino.

Daniele Dalmasso, PhD, Politecnico di Torino, DISEG, Corso Duca degli Abruzzi 24, 10129 Torino (Italy).

David Erba, Researcher, Politecnico di Torino, DISEG, Corso Duca degli Abruzzi 24, 10129 Torino (Italy).

Giovanni Vincenzo Fracastoro, Full Professor, Politecnico di Torino, DENER, Corso Duca degli Abruzzi 24, 10129 Torino. Damien Gondre, Researcher, University Lyon1, CETHIL, INSA-Lyon, 9 Rue de la Physique, 69621 Villeurbanne (France). Marco Jahn, Researcher, Fraunhofer Institute for Applied Information Technology FIT, Department of User-Centered Ubiquitous Computing, Schloss Birlinghoven, 53754 Sankt Augustin, (Germany).

Enrico Macii, Full Professor, Politecnico di Torino, DAUIN, Corso Duca degli Abruzzi 24, 10129 Torino (Italy).

Edoardo Patti, PhD, Politecnico di Torino, DAUIN, Corso Duca degli Abruzzi 24, 10129 Torino (Italy).

Anna Pellegrino, Associate Professor, Politecnico di Torino, DENER, Corso Duca degli Abruzzi 24, 10129 Torino.

Paolo Piumatti, Assistant Professor, Politecnico di Torino, DISEG, Corso Duca degli Abruzzi 24, 10129 Torino (Italy).

Ferry Pramudianto, Researcher, Fraunhofer Institute for Applied Information Technology FIT, Department of UserCentered Ubiquitous Computing, Schloss Birlinghoven, 53754 Sankt Augustin, (Germany).

Jerôme Savoyat, Researcher, University Lyon1, CETHIL, INSA-Lyon, 9 Rue de la Physique, 69621 Villeurbanne (France). Maurizio A. Spirito, Head of Research Area, Istituto Superiore Mario Boella (ISMB), Pervasive Technologies Area, Via P.C. Boggio 61, 10138 Torino (Italy).

Riccardo Tomasi, Head of Research Unit, Istituto Superiore Mario Boella (ISMB), Pervasive Technologies Area, Via P.C. Boggio 61, 10138 Torino (Italy).

Joseph Virgone, Professor, University Lyon1, CETHIL, INSA-Lyon, 9 Rue de la Physique, 69621 Villeurbanne (France).

\section{Abstract}

In this paper we report a methodology, developed in the context of Smart Energy Efficient Middleware for Public Spaces (SEEMPubS) European Project, aimed at exploiting ICT monitoring and control services to reduce energy usage and $\mathrm{CO}_{2}$ footprint in existing buildings. The approach does not require significant construction work as it is based on commercialoff-the-shelf devices and, where present, it exploits and integrates existing Building Management Systems (BMS) with new sensors and actuator networks. To make this possible, the proposed approach leverages upon the following main contributions: (a) to develop an integrated building automation and control system; (b) to implement a middleware for the energy efficient buildings domain; (c) to provide a multi-dimensional Building Information Modelling (BIM)-based visualization; (d) to raise people's awareness about energy efficiency.

The research approach adopted in the project started with the selection, as case studies, of representative test and reference rooms in modern and historical buildings chosen for having different requirements and constraints in term of sensing and control technologies. Then, according to the features of the selected rooms, the strategies to reduce the energy consumptions were defined, taking into account the potential savings related to lighting, heating, ventilation, and air conditioning (HVAC) systems and other device loads (PC, printers, etc.). The strategies include both the control of building services and devices and the monitoring of environmental conditions and energy consumption. In the paper the energy savings estimated through simulation, for both HVAC and lighting, are presented to highlight the potential of the designed system. After the implementation of the system in the demonstrator, results will be compared to the monitored data.

\section{Key words}

Interoperability, Middleware, Wireless Sensor and Actuator Networks, Building Management System, Building Information Modelling, Energy Awareness and Efficiency.

\section{Introduction}

The new era of smart Information and Communication Technology (ICT), providing real-time access to monitoring information, is bringing a new concept of energy management. Pervasive devices also enable effective control strategies 
allowing reduction in energy usage and $\mathrm{CO}_{2}$ footprint in existing public buildings without significant construction work. In particular, special attention must be paid to historical buildings where damage by extensive retrofitting must be avoided.

\subsection{The aim of SEEMPubS research}

The goals of the SEEMPubS [1] project are several and fully integrated with each other as described below:

- To develop an integrated electronic system to monitor different buildings, building services, electronic devices and operations in order to optimize and integrate all maintenance functions.

- To implement an interoperable web-based software solution for real-time energy performance monitoring and control of lighting and HVAC services through Wireless Sensor and Actuator Networks (WSAN). This is done through an integrated approach based on the LinkSmart energy-aware middleware platform [3.3]. In this way, the project is able to deploy the new solution in buildings by reusing already existing structures (e.g. proprietary sensor networks or management systems) and by integrating them into an intelligent energy management system, supported by a Graphical User Interface (GUI).

- To provide multi-dimensional visualization of parameters of building operations and data sharing from technical systems. Real-time information is collected by sensor networks detecting environmental and maintenance-oriented parameters of performance from lighting and HVAC services. The primary purpose of such a performance monitoring system is to provide facility managers and operators with the means to easily assess the current and historical performance of the building as a whole, and of its significant energy consuming systems and components. The performance monitoring system includes not only the needed sensors, wiring and data acquisition devices, but also means to calculate, display and archive resultant parameters.

- To raise people's awareness for energy efficiency in public spaces: this is a key aspect and the first step in the process of fostering sustainable and responsible behaviour with respect to energy consumption. This includes the construction of a user community portal to enable communication and information exchange among users. Such communities, consisting of users with different levels of knowledge of the energy domain, combined with ambient awareness features, have the ability to create a new energy consciousness of citizens into public spaces.

All elements are validated through an iterative methodology to ensure the gradual approximation to SEEMPubS with simultaneous consideration of all stakeholder needs. The validation phase of the project is conducted utilizing the most significant buildings in the Politecnico di Torino Campus, selected as demonstrator.

\subsection{The case studies}

The construction period of the Politecnico di Torino's campus ranges from the XVI century to the XXI century, thus including buildings very different in dimension, shell, construction characteristics and performance. The short description below helps to understand the reason of its representativeness as an example of a wide range of similar buildings in Europe.

The Valentino Castle on the river Po, seat of the Schools of Architecture is a historical site. Its origins date back to the beginning of the XVI century. A non-insulated and highly massive shell characterizes the building, and the systems have been realized taking into account the building's conservation requirements.

The Main Campus on Corso Duca degli Abruzzi, dedicated mainly to Engineering was inaugurated in November 1958. The building envelope was built with very poor thermal insulation criteria and several retrofits are being planned to increase the building's energy performance.

The Cittadella Politecnica, recently built next to the Main Campus in a former industrial area, is the Politecnico's research centre. The plan, started in 1994 and at present under completion, includes recovery of the most interesting industrial buildings, and new buildings. This campus was built adopting energy efficiency-oriented construction techniques, according to the Italian Law 10/91.

Based on the description above, it is clear that each building has specific energy needs and limitations concerning control systems which should be installed; a major challenge for the SEEMPubS project is to deal this specific deployment's details, providing a generic framework, which is able to actually impact on energy efficiency.

\subsection{The architecture of SEEMPubS Building Energy Management System}

As described previously, one of the main goals of SEEMPubS is to develop a middleware for holistic Building Energy Management Systems (BEMS). With regards to system architecture design two major requirements were identified. First, such middleware needs to support the integration of heterogeneous technologies, such as WSAN, building management systems, or smart meters. Second, the middleware must provide unified access to these technologies to support the implementation of applications that use these technologies in an interoperable way (e.g. implementation of building control 
strategies). A further requirement from the middleware perspective is that the developed components should be extensible and reusable in similar settings.

In order to cope with these requirements, the SEEMPubS consortium has decided to limit custom developments and instead employ and extend an already existing and proven technology, namely the LinkSmart Middleware.

The LinkSmart Middleware is a generic middleware for developing Ambient Intelligence (AmI) applications [2, 3]. It is the result of the FP6 European Project HYDRA [4] and it is currently employed in a large number of other EU and international research initiatives, e.g. [5, 6, 7]. AmI is characterized by making use of various different technologies and devices, which together perform ambient and intelligent computing tasks. Typical examples of AmI are smart homes or ambient assisted living projects.

LinkSmart provides a framework and software development tool for integrating heterogeneous networked devices into AmI applications. Further, LinkSmart comes with software components that provide functionality typically for AmI applications (e.g. message encryption, event management, or device discovery). The LinkSmart Middleware is applied and extended to develop a dedicated middleware for the energy efficient buildings domain - under consideration of the aforementioned requirements. In the following we describe the SEEMPubS architecture and explain to which extent LinkSmart technology is reused and extended with new functionality.

Figure 1 shows the layered architecture of the SEEMPubS system including its main components.. On the LinkSmart Proxy Layer different kinds of technologies (WSAN, BMS or smart meters) are integrated into an interoperable system. We employ the Proxy approach defined by the LinkSmart Middleware. This approach allows to use the different technologies in a unified way (cf. 4.1). A LinkSmart Proxy is a component that acts as bridge from a specific technology to the LinkSmart middleware by providing a defined service interface. In this way, proxies allow unified access to the different technologies through LinkSmart.

On the SEEMPubS Middleware Layer reside components that process data provided from the Integration Layer and provide further functionality to the Application Layer (e.g. components to execute building control strategies reside on this layer) (cf. 4.3). The Middleware Layer is conceptually separated into two parts: the LinkSmart infrastructure components and the Context Framework. The infrastructure components, namely Network Manager and Event Manager provide the core communication functionality for all LinkSmart components. The Network Manager enables network communication among devices inside a LinkSmart network. It creates an overlay P2P network that implements SOAP Tunneling as transport mechanism for web service calls [25], allowing direct communication among all devices inside a LinkSmart network, no matter if they appear behind a firewall or NAT (Network Address Translator). The Event Manager allows developers to build event-based systems, which in the case of systems dealing with sensors, is a core requirement. In SEEMPubS, the Event Manager is used to publish events at the Proxy Layer and to listen to events wherever needed, for instance at Context Framework, for further processing. The Context Framework is an extension to LinkSmart which is specifically designed for energy-efficiency applications aiming at achieving semantic interoperability. While syntactic interoperability is achieved by the Proxy Layer (by homogenizing the different standards and protocols of different technologies), semantic interoperability in SEEMPubS means to abstract from the Proxy Layer to a layer that deals with domain objects like buildings, rooms, windows, appliances, sensors, etc. Such domain information can then be used by the system for (i) end-user applications, (e.g. for visualization) and (ii) for implementing building management control strategies that work seamlessly across heterogeneous technologies. The Context Framework is described in detail in Section 4.3.2.

The Application Layer comprises end-user applications for different kinds of users (e.g. an integrated monitoring and control application for the building energy manager would be part of the Application Layer as well as applications for building occupants) (cf. 4.3). With the help of the Middleware Layer, information should be easy accessible by any kind of application. 


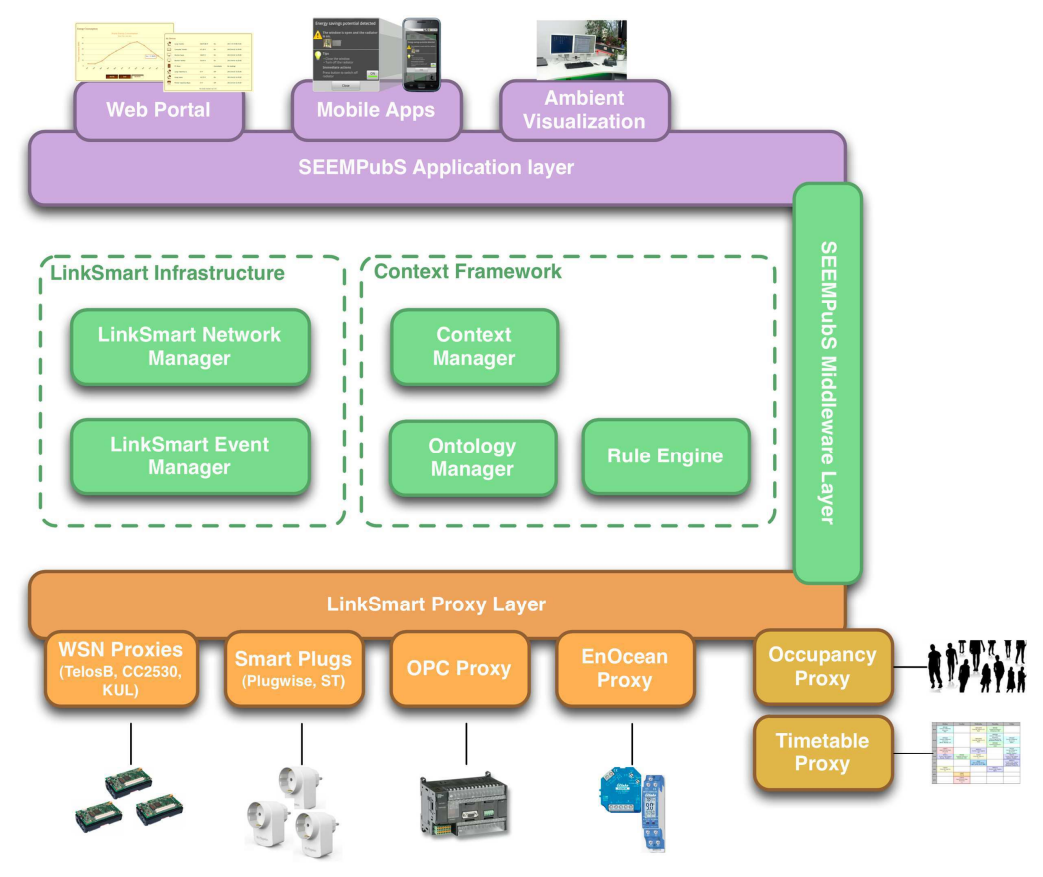

Figure 1. Layered System Architecture

\subsection{Related Work}

Several research projects are dealing with increasing energy efficiency in buildings. The FIEMSER [26] project aims at developing an energy management system (BEMS) for existing and new residential buildings. FIEMSER tries to achieve that goal by optimizing the balance between energy generated locally, energy needed from external providers and energy demanded by the building. Technically FIEMSER employs a combination of sensors and actuators from traditional PLCbased BEMS systems, connected via an OSGi+SOA REST architecture. While the goals of FIEMSER and SEEMPubS are to some extent comparable, they both aim for different domains.

Another similar project but with a different domain is 3e-House which deals with the integration of established ICT technologies in social housing in order to provide an innovative service for energy efficiency. Other projects such as SaveEnergy [27] focus on changing people's behavior through the use ICT - real time information and serious games in the case of SaveEnergy. A similar approach is implemented by the BeAware [28] project, which also aims at increasing people's energy awareness in private households.

Many other research initiatives and projects aiming at increasing energy efficient in different kinds of buildings exist, each focusing on different specific topics.. SEEMPubS tries to produce a result which can be generalizrd - namely the middleware - that can be applied in other projects dealing with energy efficiency in buildings and the aforementioned issues of interoperability. While many different projects develop custom middleware solutions, we will provide an open software that can be applied for energy efficiency applications in buildings in a general way. Using the existing LinkSmart infrastructure is supposed to support this goal.

\section{Methodology}

SEEMPubS is committed to an iterative approach following the principles of the common standard ISO 13407. The goal of this procedure is focused not only on the evolution of the applications and services, but also on the refinement of scenarios, requirements, specifications of the SEEMPubS platform and its implementation. This iterative process ensures the gradual approximation to all multidisciplinary activities involved in SEEMPubS with simultaneous consideration of all stakeholders needs as described following. 


\subsection{Integration of devices}

The strategy for smart building management and control leverages upon an ICT infrastructure made of heterogeneous monitoring and actuation devices, such as WSAN. Moreover, in order to improve backwards compatibility, the infrastructure supports wired devices work with different protocols, such as BACnet, LogWork, etc. An innovative web service oriented software infrastructure has been developed to manage heterogeneous and commercial devices. As introduced before, the LinkSmart middleware [2-4] has been adopted to provide interoperability between heterogeneous devices and networks, both existing and to be deployed. Moreover, the proposed infrastructure allows easy extension to other networks, thus representing a contribution to the opening of a market for ICT-based customized solutions integrating numerous products from different vendors. The system manages energy efficiently and WSANs are preferred to simplify the integration of new sensors into the system and also to avoid overloading of cables in historical buildings. The software infrastructure provides the following main functionalities:

- It enables the interfacing to the application layer by means of web services, through which the sensor data is read and can be used for visualization or to feed energy management policies;

- It collects environmental data coming from the sensor nodes into the local database and this data can be accessed in an asynchronous way and preserved from network failures;

- It allows the remote reconfiguration of sensor node parameters such as the sampling rates of physical quantities which are to be monitored;

- It allows the remote control of actuator devices;

- It enables interoperability among heterogeneous networks, characterized by different communication protocols, microcontrollers and sensors.

As shown in Figure 2, the software runs in a PC-Gateway (GW) and communicates directly with the heterogeneous networks. The dedicated Interface represents the lowest layer of our proposed stack, and receives information coming from various devices, regardless of the adopted communication protocols, hardware or the network topology. Hence, each network needs a specific software interface, which interprets the environmental information (e.g. temperature, humidity, etc.) and stores them in an integrated database (DB), in order to make the whole infrastructure flexible and reliable with respect to backbone network problems, since data are locally stored. The web-service layer, implemented using LinkSmart, interfaces the device networks to the web, making the remote management and control easier. Moreover it exports to the application client layer, the last in our stack, all the environmental data that was stored in the DB. At this layer, the information is available to the end-user and ready to be post-processed or to be shown via computers, tablets or smart phones. Particular emphasis was given to the possibility to reconfigure each node, changing, for instance, some parameters about power management. In this scenario, the end-user sends the new configuration via web-services to the GW and stores it in the DB. Then, the new settings will be automatically sent to the receiver mote, when it wakes up from the sleeping period, through the specific network software Interface. The configurable parameters change depending on the hardware and the Operating System running on the end node. However, using this software infrastructure, the user can choose only the right settings ignoring the real physical hardware related to the virtual device. About power management, it is worth noting that there is no standard that indicates how controls and configuration settings are to be sent to the mote via the protocol packet payload. Hence, the proposed network software Interface is hardware dependent only for the way in which these parameters are formatted. However, from the communication point of view, it is protocol standards compliant. The proposal and development of a standard for power management would be desirable but is not the subject of this project. The goal of this research, from ICT side, is to enable the communication between heterogeneous and commercial devices; this implies a certain dependence on hardware.

Particularly in this case study, we have developed different interfaces to manage respectively five commercial WSAN based on:

- Crossbow Telos rev.B [8] open source end node to monitor air temperature, relative humidity and illuminance, which exploits IEEE 802.15.4 communication protocol;

- Our customized end node built on Texas Instruments' cc2530 system on chip [9] to monitor air temperature and illuminance leveraging the ZigBee protocol;

- ST Microelectronics Smart Plug [10] commercial end node to monitor power energy consumption and to switch on/off the lighting plants, exploiting the ZigBee protocol;

- Plugwise Smart Plug [11] commercial end node to monitor power energy consumption and to switch on/off the appliances connected to the mains exploiting the ZigBee protocol;

- EnOcean protocol stack [12] commercial end nodes to monitor air temperature, relative humidity, illuminance, occupancy and to actuate heating and lighting plant respectively. 
Moreover, an interface has been developed to enable the remote communication with the Siemens Desigo BMS that exploits the BACnet protocol [13] in order to monitor and actuate Desigo wired devices.

In a nutshell, the proposed web-based infrastructure is a software that makes transparent to the end-user the underlying devices, abstracting all the information about hardware, communication protocol stack and embedded operating systems. Furthermore, the use of web-services makes the interoperability with third-party software easy.

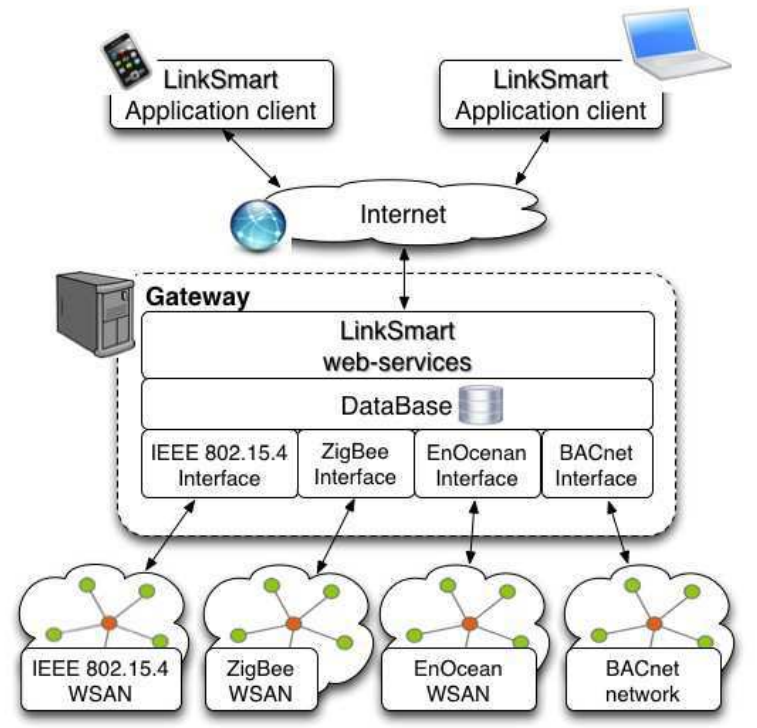

Figure 2. Software infrastructure scheme to handle heterogeneous devices

\subsection{BIM and Interoperability}

To optimize the data exchange among architecture, Mechanical Electrical and Plumbing (MEP), energy simulations and Facility Management (FM), at the beginning of the project a BIM methodology was used. For this reason, the interoperability testing described in this paragraph is an important issue of SEEMPubS, both for processing and for data visualization [14].

The BIM approach started with the creation of 3D models of each case study through Autodesk applications as shown in Figure 3. In particular Revit Architecture was used for architectural modelling and Revit MEP for the modelling of heating/cooling and lighting systems [15].

To test the interoperability between architectural modelling and lighting simulations it was necessary to realize the $3 \mathrm{D}$ models of the real rooms and their external environment. The 3D models were used to run energy simulations, which were validated through the monitored data and then used to estimate the building energy demand. In particular, Radiance [16] was used to validate the models, comparing the simulated illuminance distribution to the illuminance values measured in the corresponding rooms, while Daysim [17] was used to estimate the lighting energy demand and the savings obtained with the specific control strategies proposed for each case-study, as it allows running annual simulation for a site, accounting for the specific dynamic climate conditions.

In order to run the lighting simulations, it was not possible to import the parametric model from Revit into Radiance/Daysim directly. The software Ecotect Analysis [18] was hence used as interface to launch Radiance/Daysim. As a result, Revit was imported into Ecotect using different procedures, with the aim of finding the most appropriate.

The first trial adopted the traditional approach based on the exporting in IFC (Industry Foundation Classes) format, but it did not succeed because some elements, such as the window frames, were not exported or were displaced.

The second trial was based on the exporting from Revit by a gbXML file, but some geometrical discrepancies in the surfaces generated from the solid elements made the model unsuitable for the lighting simulation. In fact, this type of exporting involved some model simplifications that turned out to be incorrect for lighting simulations, even if the model was correctly generated and all the elements had maintained their reciprocal positions.

The third procedure was the most appropriate, both concerning modelling in Revit Architecture environment and for the analyses with Radiance and Daysim (thanks to Ecotect as a software interface), through the use of 3DStudioMax as an intermediate software (to convert the .fbx file exported from Revit in a .3ds file imported into Ecotect) which gave good results in terms of geometrical consistency of the exported model. Nevertheless, also with this procedure it was necessary to 
check the 3D models imported in Ecotect before starting the lighting simulations in Radiance and Daysim, to prevent some trivial mistakes connected to an oversight or elimination by the operator (for instance the disappearance of some parametric elements).

Once the 3D models were successfully imported into Ecotect, it was possible to proceed with the lighting simulations in Radiance and Daysim. The numerical data obtained from the simulations were graphically processed by using the Ecotect Analysis application, through the import of .dat format (coming from Radiance) or .da (coming from Daysim) and therefore shown in 3D visualization.

In a similar way it was possible to work between architectural (Revit Architecture) and thermic (Trnsys 17) models using SketchUp as additional software. Compared to the classic method, the use of the 3D model in Trnsys 17 required attention in some critical points (north orientation, window frame and wall thickness). Therefore, before starting the simulation phase, we fixed the critical points. In detail:

- The model was optimized after the importation in SketchUp, before drawing the Thermal Zones;

- The window area was adjusted. In fact, since in Revit Architecture objects are parametric, it is possible to calculate the exact area of the frame and of the glass surface for each window; this value was put in Trnsys to improve the accuracy of the simulation results;

- The problem of the wall thickness between adjacent Zones was fixed by moving the wall surface of one of the two rooms. This had as a consequence a volume increase of one or both rooms; but this does not have significant consequences on the results due to the little impact of air volume in the heat balance.

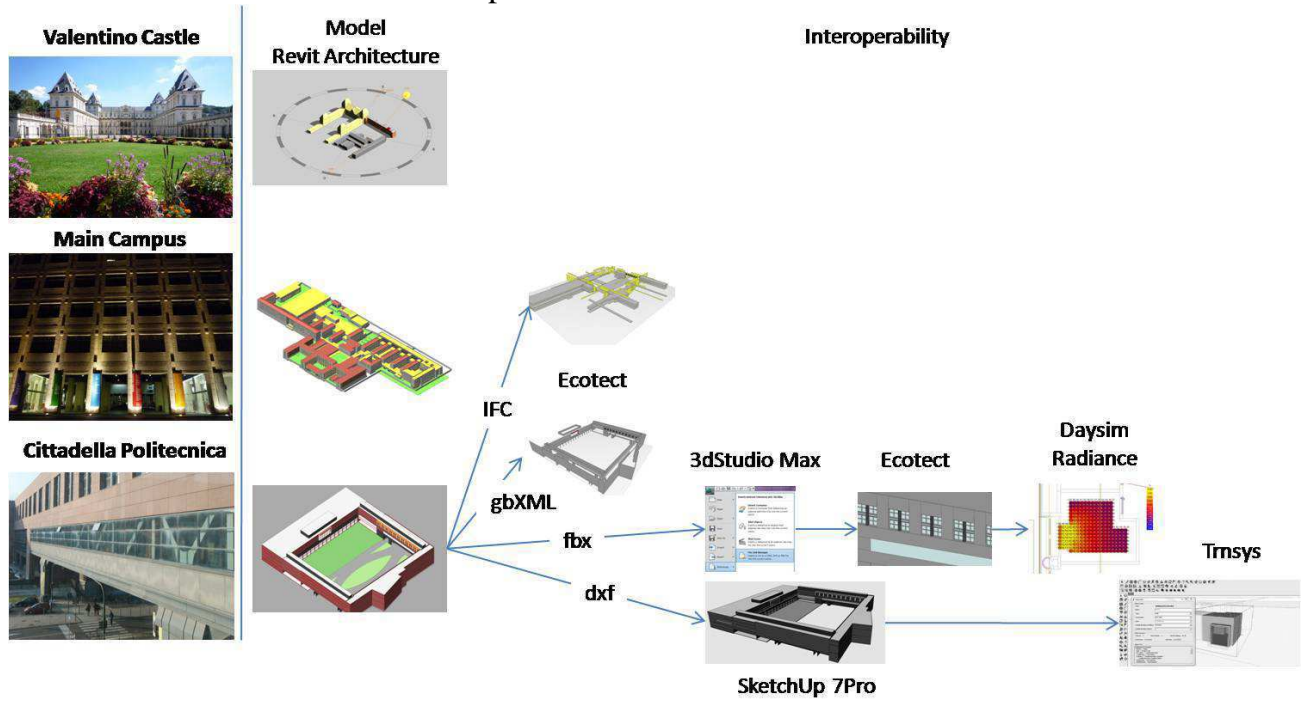

Figure 3. BIM and Interoperability as method to optimize the process

\subsection{Context awareness}

The Context Framework is part of the energy efficient middleware. From a software architecture perspective it resides between the proxy layer (as described in 3.3) and the application layer. The aim of this Context Framework is to interpret data provided by sensors and devices into semantically interoperable information that can be used by the system for (i) enduser applications (e.g. for visualization) and (ii) implementing building management control strategies that work seamlessly across heterogeneous technologies. Context-aware optimization is a generic and "complex" task, since its main goal is to adapt the building behaviour to detect and act upon a very broad and extensible set of situations. For this reason, the SEEMPubS Context Framework must provide flexible means to describe generic "situations" which are significant for energy consumption monitoring, optimization and awareness.

As an example for such a situation we define the energy-wasting situation.

An energy wasting state is defined as a situation where energy is being spent without benefit for the users. An example of energy wasting state might be represented, for instance, by a room where the window is open and the HVAC is running or a PC left idle without any use. Such information could be used to provide feedback to end-users and motivate energy-saving behaviour. Another use-case showing the advantages of semantic interoperability is the technology-independent definition of control strategies in an integrated system.

In the following we describe how the SEEMPubS Context Framework supports these concepts 


\subsubsection{From sensor data to building information}

The term semantic interoperability in our system characterizes the ability to access different kinds of devices, sensors, actuators, or systems through a unified vocabulary (cf. Ontology Manager) regardless of vendor- or technology-specific implementations, data formats, APIs, etc. Figure 4 shows how data is transformed from the actual sensor to the Context Framework. The Proxy layer is responsible for processing raw sensor data and adding some basic meta-information and forwarding it to an Event Manager. The Context Framework is responsible for associating the incoming data with a meaning.

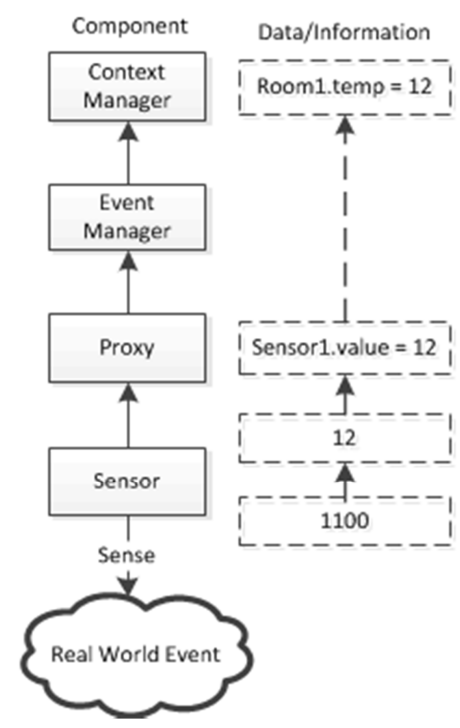

Figure 4. Abstraction of Data

\subsubsection{The Context Framework}

In this paragraph we present the implementation of the Context Framework that uses an Ontology-based knowledge repository and rule-based context recognition system that allows defining actions that should be performed upon recognition. The Ontology models the abstraction from concrete devices, sensor etc. to domain specific entities such as buildings, rooms, HVAC appliances, etc.

\section{Ontology}

The Ontology provides a taxonomy for the energy efficient buildings domain, describing a model that is used to support the development of energy efficiency applications. This model describes the (semi-) static environment of the building independently from the type of implementation or platform used in development of such applications. It models devices, sensors, actuators, rooms, and observable properties such as temperature, humidity and so on. To map from the domain to the implementation it also models software proxies, services, and events.

\section{Context Manager - Rule Engine and Entity Manager}

The Context Manager consists of two major components, (i) an Entity Manager to manage the current and past context of all entities and (ii) a Rule Engine to store and evaluate context reasoning rules.

The Rule Engine has two main roles: (i) rules are defined to recognize a situation based on the state of entities in the system and (ii) rules can also be used to define an action that should be performed upon recognition of a situation. We use the Drools [19] rule engine - a well-known rule engine for business applications - and modified the rule language allowing it to define triggers that will be used by the Context Manager to subscribe to specific sensor event which monitor the context of the entities. When a sensor event comes, the Context Manager will update the context of all entities that are associated with that event. The correlation between the sensor event and the entities is done with the help of Ontology reasoning. The drools engine will then evaluate any rules that are associated with that entity and execute the actions defined in the rules. Such action could be the invocation of an actuator.

The Entity Manager is responsible for keeping track of entities and their context attributes (e.g. temperature in Room1, humidity in Room1). An entity and its context attributes are monitored automatically once they are defined in a rule and their current state is saved. This approach enables the Entity Manager to only store the current state of the system as needed. 
By moving the storage of ever-changing context data from the Ontology to a dedicated Entity Manager we avoid performance problems in the semantic store.

In the following we provide an example that explains how the Context Framework can be used to recognize situations and perform actions accordingly.

As stated before, the Context Framework allows us to deal with domain objects. This means, we can define rules like the following: Every time the state of Window1 or Radiatorl changes check if Window1 is in state open and Radiatorl is in state on. If they are, publish to any interested component that there is an Energy Waste situation in Rooml, involving Windowl and Radiator1. The domain objects we are talking about here are Window1, Radiatorl, and Rooml.

The advantage of this approach is that for the definition of such rules, no knowledge about underlying technologies is needed. It doesn't matter if the radiator is controlled by a BACNet or EnOcean device or what kind of contact sensor is attached to Windowl

This information is stored in the Ontology and will be reasoned by the Ontology Manager. Figure 5 shows (in a simplified way, leaving out sub-classes) how the relevant information is modelled in the Ontology.

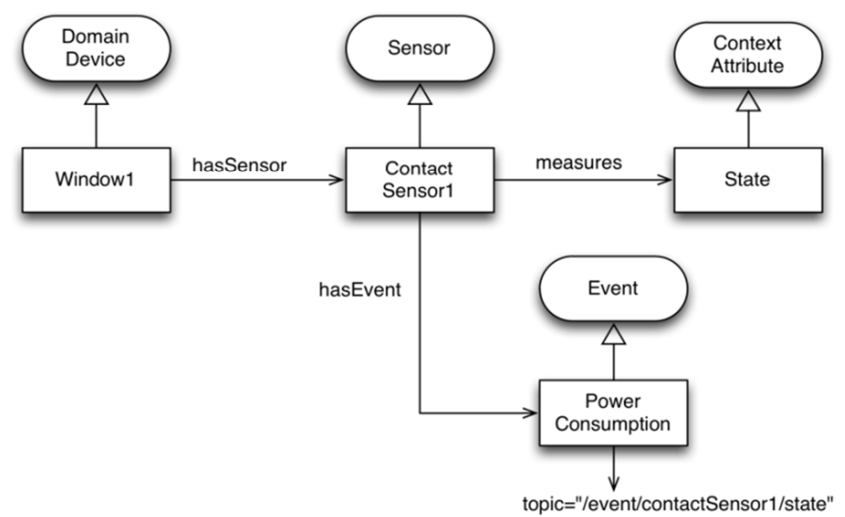

Figure 5. Ontology instantiation

Besides notification of situation changes the rule-based approach also allows for implementing control strategies, such as: Every time the presence property of Rooml changes and it is false, switch off the light in that room.

Beyond this simple example it is worth observing that presence detection, or more in general occupancy detection [12] is a complex process and can thus give more complex types of outputs rather than "true/false" presence information. Therefore, occupancy values might have been evaluated beforehand by other rules or by dedicated components, for instance an Occupancy Proxy that takes care of optimizing occupancy recognition by using multiple input sources and adding probabilities to occupancy states.

As for recognition the Context Framework also handles the invocation of an actuator for switching the light. Therefore, the Context Manager consults the Ontology to find the respective actuator. A standardized Proxy interface allows executing the correct service via the proxy that communicates with this actuator.

\subsection{Control rules, control strategies (middleware)}

Since the project aims at controlling HVAC and lighting systems using temperature, luminance and occupancy sensors, new control rules must be designed and tested in order to properly balance energy savings and comfort issues [20]. The thermal inertia of buildings leads to a low responsiveness of the HVAC system, which requires an anticipation of possible future use of a room in order to meet comfort requirement within a reasonable time when occupancy is detected. The test cases have been modelled in Daysim (lighting simulations) and Trnsys (thermal simulations) and several control rules have been implemented and tested. According to the information on the rooms features, achieved during the first phase of the project, some lighting control strategies were initially proposed. In particular, for spaces with high daylight availability and medium/high users absence probability, a combination of daylight harvesting and occupancy detection was proposed as lighting control strategies, while, for spaces with low daylight penetration, only occupancy detection was supposed to be used. In any case users are allowed to override the system with manual control. Thermal control strategies are guided by the maintenance or improvement of user comfort combined to the best energy savings. As the uncertainty in occupancy schedules is detrimental to savings and/or comfort, a difference has been made between personal offices where presence detection is the only available information, and common rooms (meeting rooms or classrooms for instance) where users have to book the room, which allows strategies based on anticipation. 
Another difficulty is to evaluate the time necessary to reach the set point temperature when preheating is planned. It depends on the inertia of the building but also on the weather conditions and the indoor temperature. A precise calculation of this "recovery time" would lead to the best compromise in terms of energy savings and user comfort. Figure 6 below displays the main control rules considered for both lighting and HVAC systems.

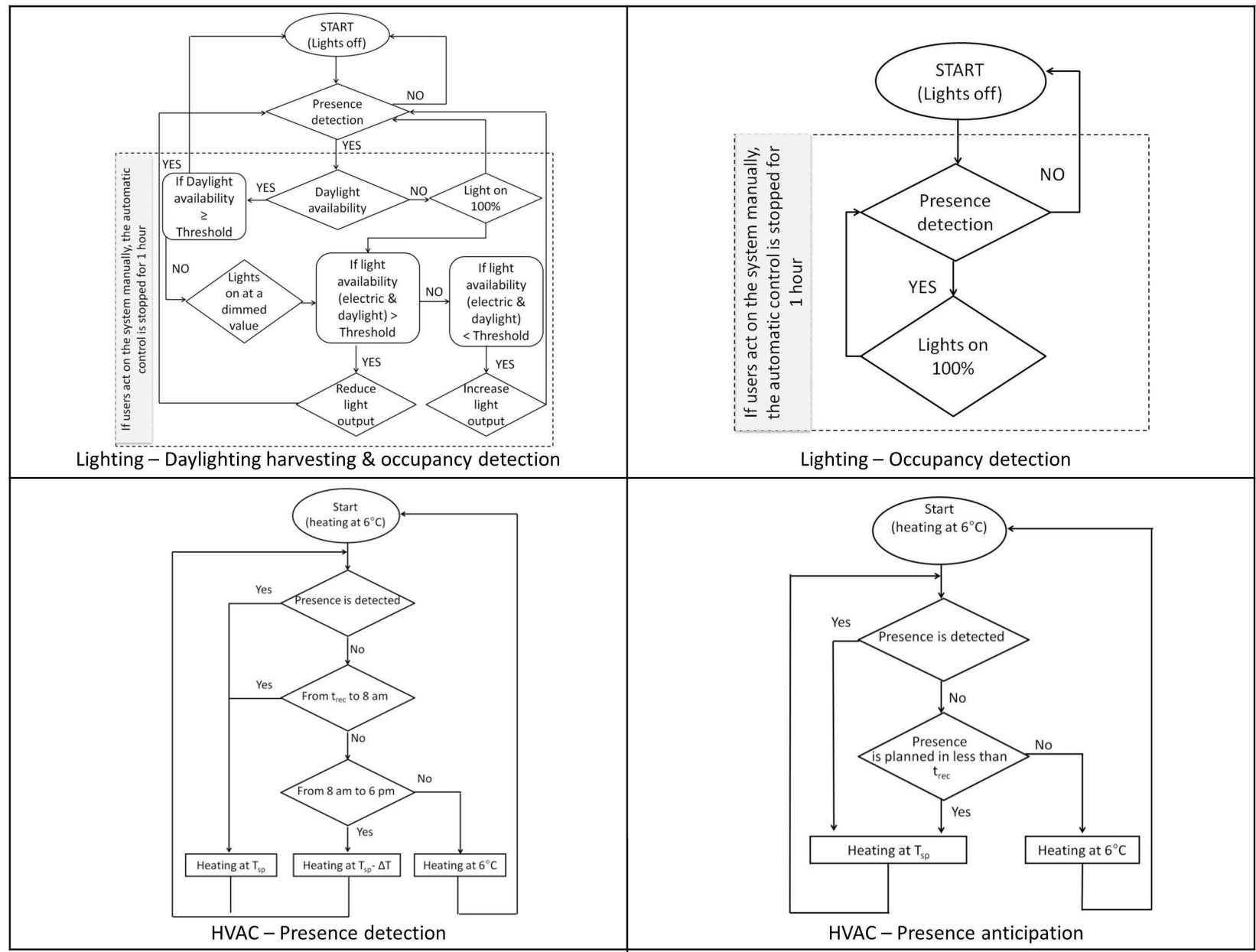

Figure 6. Control rules considered for lighting and HVAC systems (Tsp: setpoint temperature, $t_{\text {rec: }}$ : recovery time).

\section{Results}

SEEMPubS is still under way but first simulation results are already available. Simulations are part of a preliminary work that aims at optimizing energy conservation strategies that are implemented in the demonstrator. It is already planned to compare numerical results with experimental results as soon as results are available. The following paragraphs presents some simulation results of the ongoing work on energy saving in the HVAC and lighting systems.

\subsection{Estimated energy savings, heating and cooling}

This paragraphs aims at presenting detailed results for one of the six case studies. The test office is part of the Valentino Castle, the historical building made of heavy walls without thermal insulation. Its energy consumption is high and its thermal responsiveness is very low.

As savings strategies are based on an optimization of vacancy periods, results heavily depend on occupancy hypothesis used in the simulations. Two different kind of occupation profiles are used in the simulation. The "theoretical occupancy" starts at $8 \mathrm{AM}$ and ends at $6 \mathrm{PM}$ (ten hours a day, five days a week, which means fifty hours a week) and corresponds to the time range when occupancy probability is high. In the presence detection algorithm, the theoretical occupation profile does not provide a lot of details since it is hard to forecast the actual occupancy, while in the presence anticipation algorithm, the 
theoretical occupancy profile is detailed as much as possible since it is used for classrooms and meeting rooms where it is easy to know in advance what the future occupation should be. The "real occupancy" is directly linked to the occupancy detection sensor but in the simulation it is set as fixed ranges which include two full occupancy days (on Mondays and Fridays) with a one-hour lunch break and two half occupancy days (on Tuesdays and Wednesdays) with a five-hour occupancy divided into three periods ( $8 \mathrm{AM}-10 \mathrm{AM}, 1 \mathrm{PM}-2 \mathrm{PM}, 4 \mathrm{PM}-6 \mathrm{PM}$ ). No occupancy at all is considered on Thursdays. For instance, it may represent a day off, a seminar day or a day out of office. With regards to this simulation hypothesis, the real occupancy profile used in the simulation represents $56 \%$ of the theoretical occupancy.

As the presence anticipation algorithm was originally designed for common rooms such as classrooms or meeting rooms, results discussed later will mainly focus on a the presence detection algorithm.

In order to optimize the restarting time of the HVAC system in the early morning or after a period of vacancy, a study was carried out in order to characterize the building inertia. The idea was to find the best correlation between the time $\Delta \tau$ necessary to reach the set point temperature (referred to as recovery time later in the text) and the ambient conditions such as the outdoor temperature, the indoor temperature $T_{i n}$ and the setpoint temperature $T_{s p}$. Restarting curves have been analysed for 154 mornings of a yearly simulation. Monday mornings were analysed separately since the thermal behaviour of the building is different after two unheated days.

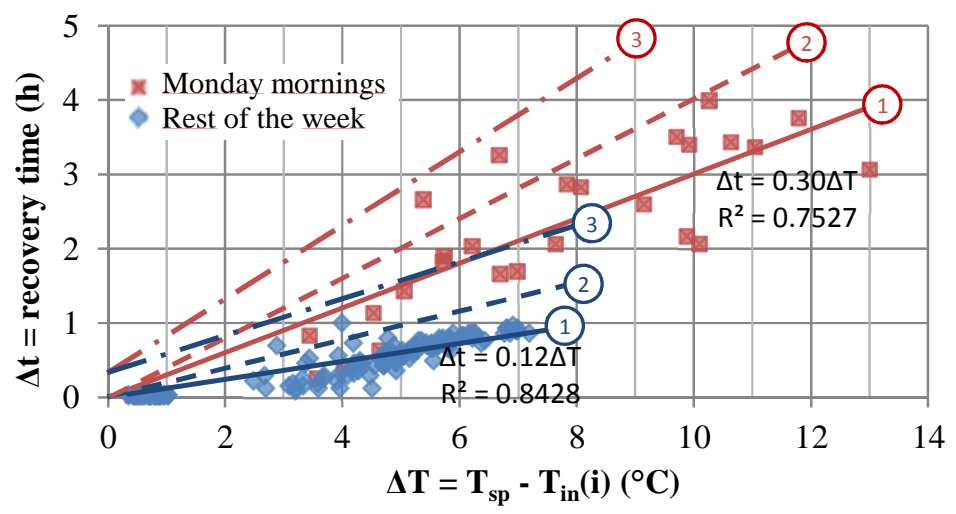

Figure 7. Recovery time as a function of the temperature difference $\Delta t=T_{s p}-T_{i n}(i)$ during the heating period

The best correlations linked the recovery time to the temperature difference between set point and indoor temperature at the restarting time. As this very simple linear equation (lines 1 on Figure 7 and Table 1 below) is not sufficient to properly describe the building behaviour, its direct use leads to good energy savings but also leads to discrepancy of user comfort (see Figure 8 below), due to the underestimation of the recovery time for about half of the time. In order to optimize the balance between energy savings and user comfort, two updated correlations are used (lines 2 in Figure7 and Table 1). As it is not sufficient to improve comfort as much as expected, a second set of updated correlations is used (lines 3 in Figure 7 and Table 1).

Energy savings values are given in percentage of savings compared to simulation results of the actual control rules used in the case study (reference room). The main differences with the test room lie in the absence of presence detection and in the use of only two set-point temperatures (one at night, and another one during working hours). As indoor temperatures may be higher in the reference room than in the test room (especially during working hours when occupancy is not "detected"), non-negligible heat transfers occur from the reference room to the test room. This leads to an over-estimation of the reference room consumption and an under-estimation of the test room consumption. Results can then be flattering and ...false! In order to calculate the consumption that would actually be observed if the whole building was equipped with the same control system, two simulations are run separately: one with the actual conservation strategy (reference), and another one with the proposed conservation strategy (test). Comfort analysis is based on the operative temperature distribution in the first hour of occupancy (8AM - 9AM) when occupancy is "detected" (figure 8).Results are very close to the present situation in terms of comfort and this strategy allows a $14.6 \%$ reduction on the overall annual energy demand (Table 1).

Table 1. Correlations used in the three test cases for calculation of the HVAC restarting time and estimation of associated energy savings 


\begin{tabular}{|l|l|c|c|c|}
\hline & Correlations used for estimation of recovery time & \multicolumn{3}{|c|}{ Energy savings (\%) } \\
\cline { 3 - 5 } & \multicolumn{1}{|c|}{ Overall } & Heating & Cooling \\
\hline \multirow{2}{*}{ Test case 1 } & $\Delta t=0.30\left(T_{s p}-T_{i n}(i)\right)$ (Monday morning) & $22.1 \%$ & $25.7 \%$ & $9.2 \%$ \\
\hline \multirow{2}{*}{ Test case 2 } & $\Delta t=0.12\left(T_{s p}-T_{i n}(i)\right)$ (rest of the week) & & & \\
\hline \multirow{2}{*}{ Test case 3 } & $\Delta t=0.40\left(T_{s p}-T_{i n}(i)\right)$ (Monday morning) & $19.6 \%$ & $22.5 \%$ & $9.4 \%$ \\
\hline
\end{tabular}

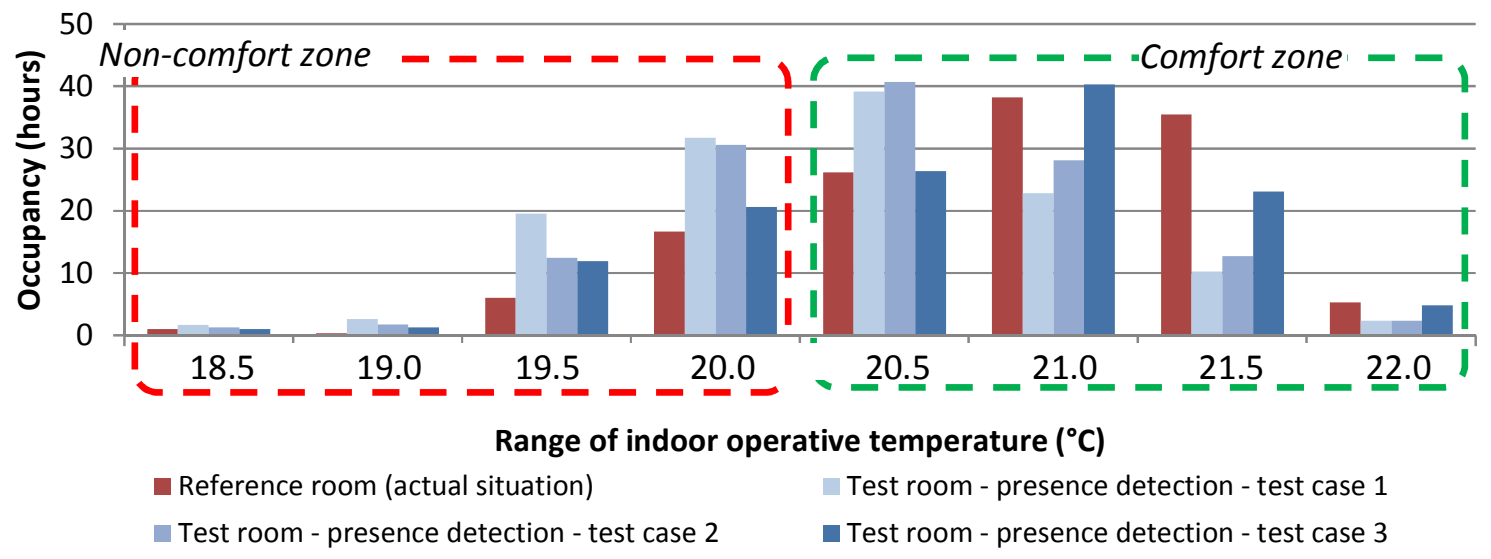

Figure 8. Indoor operative temperature distribution during heating season between 8:00 and 9:00 in the office of Valentino Castle for different control strategies

These first simulation results show that the estimation of the recovery time can be improved in the future. The more accurate the estimation of recovery time is, the less energy is needed in order to reach a given comfort level (and the more efficient are the control rules). Nevertheless the recovery time heavily depends on the building characteristics, but also on the HVAC system specifications (water and air flow rates and temperatures of the fan coils unit) that are difficult to know with precision. It then requires to fit simulation results with monitoring results in order to find the most likely HVAC specifications. These control rules will therefore require a lot of data and studies to be extended to new buildings with different characteristics. That is the reason why we are currently studying the feasibility of developing a self-learning system that would itself calculate an estimation of the recovery time, based on data collection of the past few days or months. It would avoid thermal inertia calculation (and associated data collection) since the system would calculate it itself. An algorithm based on presence anticipation also requires the recovery time calculation and also needs to be linked to a schedules DB. Simulation on the same office showed slightly better results in terms of comfort but higher energy consumption for presence anticipation strategy. It is probably due to the high inertia of the building, which leads to slow temperature variation when the HVAC system is turned off. For vacancy period shorter than a couple of hours, the presence detection strategy does not call for heating or cooling while the presence anticipation strategy restarts the HVAC systems in advance, which leads to higher power consumption. Presence anticipation strategy is then probably more relevant for low occupancy rooms in low inertia buildings. Results showed that it is possible to save up to $22.6 \%$ (32.5\% on heating and 3.9 $\%$ on cooling) in the classrooms case study.

The development of HVAC strategies will continue in two perspectives: extending detailed analysis of predictable energy savings to similar spaces (offices) of other types of buildings (Main Campus and Cittadella Politecnica), and analysing the results obtained from prototypes that will be installed in the six test rooms

\subsection{Estimated energy savings, lighting}

To assess the effectiveness of the new lighting control solutions the energy savings achievable with respect to the actual reference situation (manual lighting control), were calculated from the estimated total annual electric lighting energy consumptions. The total annual energy used for lighting depends on different aspects, mainly related to the indoor daylight availability, the building usage (occupancy profile and lighting requirements), the users' behaviour in terms of interaction 
with the lighting systems, and the lighting plant characteristics. The simulations carried out with Daysim are based on the annual weather data for Torino, on the actual offices occupancy profile (monitored during le previous phase of analysis) and lighting requirement (target illuminance of $500 \mathrm{lux}$ ), on the plant power consumption including electricity consumption associated to the control devices, and on stochastic user behaviour models implemented in Daysim to mimic how building occupants interact with manual controls of lighting plants or shading devices. In particular simulations were initially carried out considering a "mixed users' behaviour" that means users are partly active and partly passive with respect to the use of electric lighting and blinds. Active user is a user who operates the electric lighting in relation to ambient daylight condition, opens blinds in the morning, and partly closes them to avoid visual discomfort, while a passive user is a user who keeps the electric lighting switched on throughout the working day and keeps the blinds lowered throughout the year.

Simulations and comparisons between energy demand with the existing control system (manual control) and the new proposed lighting control rules were carried out for all rooms selected as case studies for the SEEMPubS project. The calculated energy saving ranges between $5 \%$ and $16 \%$ for offices with manually controlled blinds, while it rises up to $30 \%$ when both electric lighting and blinds are automatically controlled. Energy savings are calculated as relative difference between the simulated consumption of existing reference control solution (manual control) and the new proposed lighting control rules.

Analysing the results achieved from the lighting simulations, whose goal was to verify the effectiveness, in terms of energy saving, of the proposed control strategies for each room, it was observed that, in most of the cases, obtained energy savings were below the expectations. The initially proposed control strategies were mainly based on switching lights on and off as a consequence of users presence detected by the occupancy sensors, and on the automatic dimming of lights as a consequence of daylight availability. To optimise the energy saving results it was decided to simulate a control rule which provided for users to turn the lights on when the luminous environment is perceived too dark and for occupancy sensors to switch them off automatically when users absence is detected. When switched on, in rooms with high daylight availability, lights are dimmed in order to maintain the target illuminance on the working plane. Figure 9 shows the new proposed control rules for lighting.

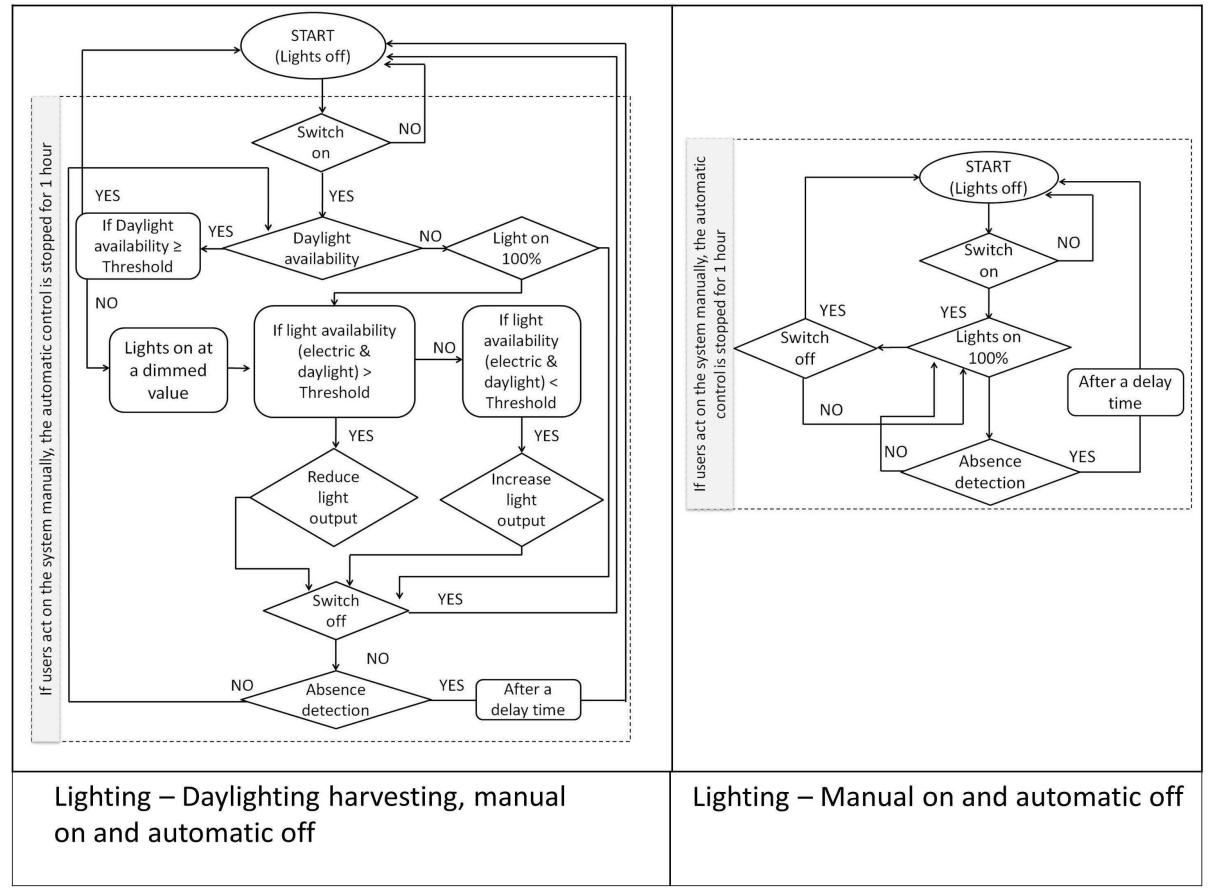

Figure 9. Optimized control rules for lighting.

The reduction of electric lighting consumption for the different rooms now ranges between $27 \%$ and $48 \%$ for rooms with high daylight availability and it reaches $13 \%$ for the open plan office where only switching off based on occupancy detection was considered. The last hypothesis that was checked was based on a different user behaviour: as it is supposed that the SEEMPubS platform should increase people's awareness by means of a web-based tool to directly interact and communicate information to users, the last set of simulations were carried out considering an "active user", that is a user who behaves consistently with the actual indoor and outdoor environmental conditions [21]. The energy savings obtained 
with the described assumptions rise to a maximum of approximately $70 \%$. In this last case both savings due to optimised control of lighting and improvement of user behaviour are considered.

A summary of the calculated electric energy savings for lighting is presented in Table 2.

Table 2. Summary of the energy savings obtained for different control rules and user behaviour

\begin{tabular}{|c|c|c|c|}
\hline \multicolumn{4}{|c|}{ Energy savings [\%] [(Energy $y_{\text {reference control rules }}-$ Energy $\left._{\text {proposed control rules }}\right) /$ Energy proposed control rules $\left.)\right]$} \\
\hline & $\begin{array}{l}\text { Auto on/off }+\operatorname{dimm} \text { (mixed } \\
\text { user behav.) }\end{array}$ & $\begin{array}{l}\text { Manual on + auto off + } \\
\text { dimm } \\
\text { (mixed user behav.) }\end{array}$ & $\begin{array}{l}\text { Manual on + auto off + } \\
\text { dimm } \\
\text { (active user behav.) }\end{array}$ \\
\hline DAUIN single office & $16 \%$ & $39 \%$ & $71 \%$ \\
\hline ADMINISTRATIVE office & $6 \%$ & $27 \%$ & $70 \%$ \\
\hline DITER office & $30 \%$ & $48 \%$ & $64 \%$ \\
\hline & $\begin{array}{l}\text { Auto on/off } \\
\text { (mixed user behav.) }\end{array}$ & $\begin{array}{l}\text { Manual on + auto off } \\
\text { (mixed user behav.) }\end{array}$ & $\begin{array}{l}\text { Manual on + auto off } \\
\text { (active user behav.) }\end{array}$ \\
\hline DAUIN open plan office & $5 \%$ & $13 \%$ & $14 \%$ \\
\hline
\end{tabular}

The technical architecture used for lighting control does not differ from previous commercial solutions. The type of sensors used in Seempubs project (photosensors and occupancy sensors) are commonly used to control lighting and the sensors layout as well does not differ from standard solutions. Control strategies instead, have been analysed in order to find the most effective solution by comparing possible alternative control logics for each room. In most existing buildings the same control logics are applied in rooms with very different features, in this way reducing the saving potentials of the automatic control system.

This is clearly highlighted by the savings results obtained by comparing, through simulation, the lighting control solution typically proposed by many commercial products and adopted in many offices (auto on/off and dimming) with control logics, based on occupancy as well as daylighting availability, but with a different degree of manual and automatic control (eg. savings rising for a single room from $16 \%$ to $39 \%$ ).

Furthermore, the SEEMPubS middleware, unlike common commercial systems, allows the monitoring and recording of both lighting energy, environmental lighting conditions and plant status, thus allowing facility managers to check the system operation and in case of fault or ineffective operation to quickly intervene to recalibrate the system. It is in fact commonly known that a system behavior not meeting users' expectations is one of the main cause of automatic control systems rejection.

Results obtained with the simulation carried out in this phase of SEEMPubS project are consistent with in case of active user behaviour or grater than the savings achieved with automatic lighting control in other similar research activates [22] [23] [24].

\section{Conclusion}

In this study we tested how ICT makes it possible to integrate different processes, applications, systems and technologies in order to reduce energy consumption. First of all, to obtain a smart building management and control leverages, we defined an ICT infrastructure made of heterogeneous monitoring and actuation devices and we developed the LinkSmart middleware to collect environmental data coming from the sensor nodes in a local DB. Then we proposed a web-based infrastructure to make transparent to the end-user the underlying devices, abstracting all the information about hardware, communication protocol stack and embedded operating system. Moreover, we set a Context Framework that uses an Ontology-based knowledge repository and rule-based context recognition system to allow the definition of actions that should be performed upon recognition. Finally, we used BIM and interoperability to process and visualize all data essential for energy simulations and for FM. Our results concern control rules and test on HVAC and lighting systems using temperature, luminance and occupancy sensors, in order to properly balance energy savings and comfort issues. Future work should optimize the increase in people's awareness by a web-based tool to directly interact and communicate information to users.

\section{Acknowledgments}

The research is funded by EU, FP7 Collaborative project - 2010: Smart Energy Efficient Middleware for Public Spaces. 


\section{References}

[1] http://seempubs.polito.it/. Last visited 06/2012.

[2] Eisenhauer, M., Rosengren, P., Antolin, P. A Development Platform for Integrating Wireless Devices and Sensors into Ambient Intelligence Systems.Sensor, Mesh and Ad Hoc Communications and Networks Workshops, 2009. SECON Workshops '09. 6th Annual IEEE Communications Society Conference on, vol., no., pp.1-3, 22-26 June 2009.

[3] Eisenhauer, M., Jahn, M., Pramudianto, F., Sabol, T., Hreno, J. 2011. Towards a generic Middleware for developing Ambient Intelligence Applications. 2nd Workshop on eeBuildings Data Models at CIB W078 - W102, 26-28 October 2011, Sophia Antipolis.

[4] HYDRA Project. http://www.hydramiddleware.eu/. Last visited 04/2012. European Commission permalink: http://cordis.europa.eu/projects/rcn/79422_en.html.

[5] SEAM4US Project - Sustainable Energy mAnageMent for Underground Stations.http://seam4us.eu/. Last visited 06/2012. European Commission permalink: http://cordis.europa.eu/projects/rcn/100734_en.html.

[6] Adapt4EE Project. http://www.adapt4ee.eu. Last visited 06/2012. European Commission permalink: http://cordis.europa.eu/projects/rcn/100798_en.html.

[7] ebbits Project- Business-Based Internet of Things and Services.http: //www.ebbits-project.eu. Last visited 06/2012.European Commission permalink: http://cordis.europa.eu/projects/rcn/96598_en.html

[8] Crossbow Telos rev B datasheet: http://bullseye.xbow.com:81/Products/Product_pdf_files/Wireless_pdf/TelosB_ Datasheet.pdf. Last visited 06/2012.

[9] Texas Instruments' CC2530 Datasheet, http://www.ti.com/lit/ug/swru191b/swru191b.pdf. Last visited 06/2012.

[10] ST Microelectronics Smart Plug: http://www.st.com/internet/evalboard/product/250409.jsp. Last visited 06/2012.

[11] Plugwise: Smart wireless solutions for energy saving, energy monitoring and switching. http://www.plugwise.com.

Last visited06/2012.

[12] EnOcean Alliance. http://www.enocean-alliance.org/en/home/?rnd=1. Last visited 06/2012.

[13] BACnet protocol. http://www.bacnet.org/. Last visited 06/2012.

[14] Osello, A. (2012). The future of drawing with BIM for Engineers and Architects. In Osello, A., Acquaviva, A., Macii, E., Patti, E., Aghemo, C., Blaso, L., Fracastoro, G., Pellegrino, A., Dalmasso, D., Piumatti, P., Savoyat, J., (Ed), Data standardization in an interdisciplinary research, pp.189-253. Palermo: Flaccovio.

[15] http://www.autodesk.co.uk/adsk/servlet/pc/index?siteID=452932\&id=14645193. Last visited 06/2012.

[16] ] Larson G. W., Shakespeare R. A. (1998). Rendering with Radiance. The Art and Science of Lighting Visualization. Morgan Kaufmann Publishers, ISBN 1-55860-499-5

[17] Reinhart C F (2001). Daylight Availability and Manual Lighting Control in Office Buildings " Simulation Studies and Analysis of Measurements. Ph.D. thesis, Technical University of Karlsruhe, Faculty of Architecture, October 2001.

[18] Ecotect Analysis official website: http://usa.autodesk.com/ecotect-analysis/. Last visited 06/2012.

[19] https://www.jboss.org/drools/. Last visited 06/2012.

[20] G.V. Fracastoro, J. Virgone, C. Aghemo, A. Pellegrino, L. Blaso, J. Savoyat, K. Johannes (2012). Energy efficiency in public buildings through ICT based control and monitoring system. In: 5th International Building Physics Conference (IBPC): "The Role of Building Physic in Resolving Carbon Reduction Challenge and Promoting Human Health in Buildings, Kyoto, Japan, May 28-31, 2012 pp. $825-832$.

[21] Reinhart C. F. 2004. Lightswitch-2002: a model for manual and automated control of electric lighting and blinds. In: Solar Energy, 77 (2004), pp. 15-28.

[22] D. H. W. Li, K. L. Cheung, S. L. Wong, T. N. T. Lam (2010). An analysis of energy-efficient light fittings and controls. In: Applied Energy, 87 (2010), pp. 558-567

[23] E. S. Lee, S. E. Selkowitz (2006). The new York Times Headquarters daylighting mockup: Monitored performance of the daylighting control system. In: Energy and buildings 38 (2006) pp. 914 - 929

[24]G. Y. Yun, H. Kim, J. T. Kim (2012). Effects of occupancy and lighting use patterns on lighting energy consumption. In energy and buildings 46 (2012) pp. $152-158$.

[25] F. Milagro, P. Antolin, P. Kool, P. Rosengren, and M. Ahls’ [11] J. M“* uller, M. Jentsch, C. Kray, and A. Kr“ en. SOAP tunnel through a P2P network of physical devices. In Internet of Things Workshop, Sophia Antopolis, Sep, 2008.

[26] http://www.fiemser.eu/. Last visited 09/2012.

[27] http://www.ict4saveenergy.eu/. Last visited 09/2012.

[28] http://www.energyawareness.eu/beaware/. Last visited 09/2012. 